\title{
Perturbative Computation of the Gluonic Effective Action via Polyakov's World-Line Path Integral
}

\author{
S. D. Avramis $\stackrel{*}{*}$ A. I. Karanikas ${ }^{\dagger}$ and C. N. Ktorides $\stackrel{\ddagger}{\ddagger}$ \\ University of Athens, Physics Department \\ Nuclear 83 Particle Physics Section \\ Panepistimioupolis \\ GR 157-71, Athens, Greece
}

\begin{abstract}
The Polyakov world-line path integral describing the propagation of gluon field quanta is constructed by employing the background gauge fixing method and is subsequently applied to analytically compute the divergent terms of the one (gluonic) loop effective action to fourth order in perturbation theory. The merits of the proposed approach is that, to a given order, it reduces to performing two integrations, one over a set of Grassmann and one over a set of Feynman-type parameters through which one manages to accommodate all Feynman diagrams entering the computation at once.
\end{abstract}

\footnotetext{
*e-mail: savramis@cc.uoa.gr

†e-mail: akaranik@cc.uoa.gr

$\ddagger$ e-mail: cktorid@cc.uoa.gr
} 


\section{Introduction}

Improved methods, in comparison to the Feynman diagrammatic ones, for expediting perturbative calculations in QCD have emerged, within the last decade or so, through the adoption of first-quantization-based approaches. The latter involve either string or world-line agents through which one describes the field theoretical system. The original efforts in this direction were string inspired and were based on realizations, made in the late 1980's [1-5], regarding the relation between string and non-abelian gauge field theories in the limit of an infinite string tension. Following their own involvement in such studies, Bern and Kosower [6] established a set of rules expediting efficient, one loop, computations in non-abelian gauge theories. Through them one could encompass contributions of a host of Feynman diagrams at once. Further extensions of the stringinspired approach were subsequently carried out in Refs. [7-12].

World-line based methodologies aiming at the same goals soon followed through the work of Strassler [13] who proposed suitably defined (one-dimensional) path-integrals for the various quantum field systems he considered. Extensive use was made of supersymmetric one-dimensional particle coordinates [14-16] 'living' on the paths. The end result was the accomplishment of the full reproduction of the Bern-Kosower rules. Strassler's approach was further pursued in Refs. [17-19], where computations pertaining to multi-loop configurations in QED as well as effective actions involving constant, external (chromo)electric and (chromo)magnetic fields were undertaken.

Now, the world-line casting of relativistic quantum systems is an old story, which goes back to Fock [20], Feynman [21] and Schwinger [22]. Notable, relevant contributions followed by several authors [23-26], the latter of which sparked our original interest in the subject $[27,28]$. What particularly attracted our attention was the geometrical setting underlying the construction of Polyakov's (world-line) path integral. Within this context we pursued the problem of tracing the field theory origins of Polyakov's spin factor, introduced by him to properly account for the propagation of a free, spin- $1 / 2$ particlelike entity on a closed (Euclidean) space-time contour. In Ref. [28] we established, via a well-defined procedure, the emergence of the spin factor through the recasting of the, spin-1/2, matter field sector of a gauge field theory from a functional to a (worldline) path-integral, entering as an appropriate weight to account for spin. At the same time, the field theoretical interaction term円 $\bar{\psi} \gamma_{\mu} \psi A^{\mu}$ is replaced by a 'factorized' Wilson line (or loop) which accounts for the effect of the gauge field on the world-line paths, equivalently describes its interaction with the matter particles. Our first applications turned in the direction of considering situations when it is justified to set the spin factor to unity -an occurrence which facilitates a factorization of the infra-red sector of the gauge field theory, in its perturbative version [29].

More recently, we have tested the possible merits stemming from the aformentioned disentanglement between spin-factor and Wilson line(loop), inherent in the Polyakov

\footnotetext{
${ }^{1}$ The interaction term is displayed generically, independent as to whether the gauge theory is abelian or non-abelian.
} 
(world-line) path integral for spin-1/2 particle-like entities, as far as the task of facilitating effective, perturbative computations in QCD is concerned [30,31]. In the first of these papers the emphasis was placed on extending the world-line methodology to open fermionic lines. At the same time we established a procedure by which Strassler's path integral expression for spin-1/2 matter particles, entering an (interacting) gauge field theory and which contains the term $\sigma \cdot F$ in the action, can be recast into the Polyakov form which carries, in its place, the spin-factor. Our subsequent manipulations were expedited by the presence of the spin factor and produced, as a bonus, the following physical picture: Non-trivial spin-factor contributions come precisely at those points where a gauge field quantum is emitted or absorbed by the fermionic world-line path. Moreover, each such occurence signifies the presence of a derivative discontinuity on the path as a four-momentum $k_{\mu}$ is locally injected. This is, indeed, a nice intuitive picture as it connects the mathematical fact pertaining to the dominance of non-differentiable paths on the one hand, with the physical occurence of emission and absorption of quanta on the other. In the second paper we focused our attention on the more pragmatic goal of developing algorithms, always for spin-1/2 particle (open) world-lines, which lead to efficient perturbative computations in QCD pertaining to Green's functions and amplitudes. Two different alternatives were arrived at according to whether the execution of the particle path-integral preceeds or follows the considerations involving the Wilson line (loop): (a) The Feynman diagrammatic logic is directly visible and comprehesively dealt with, (b) a novel organization of the perturbative expansion is achieved which retains the space-time description all the way. Strategy (a) leads to a neat organization of the resulting perturbative expression which reduces the computation to two straightforward steps. The first pertains, basically, to the spin-factor and amounts to a simple integration over a set of Grassmann variables, as many in number as the perturbative order considered. The second integration is over a set of proper time parameters inherited from the expansion of the Wilson exponential. Possible practical merits of alternative (b) constitute an open issue.

Encouraged by the fact that the Polyakov path-integral for spin- $1 / 2$ particle entities leads to computational procedures which, both logistically and intuitively, seem to present advantages of their own, we undertake, in the present paper, the task of extending the application of the relevant methodology to the gluonic sector of QCD. Such an effort entails, among other things, the determination of the spin factor pertaining to the propagation of a spin-1 particle like entity. This issue will be confronted in Section 2 where we consider the pure gauge field sector of a Yang-Mills system and utilize techniques associated with the background gauge field fixing procedure. Focusing on effective action terms at the one gluon loop level we proceed, in Section 3, to produce a master expression furnishing the $M t h$ perturbative order contribution. The overall structure of these terms corresponds to a gluonic world-line loop on which 'vertex operators', in the form of plane waves are attached. As in our previous work [31], pertaining to open fermionic world-lines, the overall calculation reduces to an integration over a Grassmann set of parameters followed by one over a set of Feynman-type parameters. The number of variables for each of the two sets is fixed by the perturbative order, while 
the execution of the integrals themselves is a fairly straightforward matter, as witnessed by the applications worked out in Section 4. There, the master formulas are used in order to compute the divergent parts of the 2nd, 3rd and 4th order one gluon loop contributions to the effective action. From the specific manipulations it becomes totally apparent how the world-line configuration accommodates, to a given order, the totality of the contributing Feynman diagrams via straightforward permutations of the parameters. As already mentioned, our analytic computations in this section are restricted to the divergent contributions (furnishing renormalization factors) which can be readily identified and isolated from the full expression to each given order. In this connection, it can be easily surmised from our master expressions that no divergent terms make their appearance above the 4th order, an occurrence that complies with the renormalizability of the theory. As far as the finite contributions are concerned, what we can say at this stage is that we are in the process of finalizing tests of relevant numerical procedures that have been devised for coping directly with their computation. We intend to report on this matter in the near future [32]. Finally, in Section 5 we summarize our findings and formulate our conclusions, while in an Appendix we trace the main steps involved in bringing the spin-factor to its final, ready to apply form.

\section{Polyakov World-Line Path Integral for the Gluon Sector of QCD}

The successful trancription of the fermionic sector of a gauge field theory into its Polyakov path integral| ${ }^{2}$ form utilizes the fact that the corresponding functional integral is of a gaussian type [28]. For the gluon sector, of course, such is not the case. Following Refs. $[13,19]$ we proceed by employing the background gauge fixing procedure according to which the gauge field $A_{\mu}$ splits into a dynamical component, to be denoted by $\alpha_{\mu}$, and a background field $B_{\mu}$. Given that we shall restrict, in the present paper, ourselves to the computation of effective action terms, the background field will be considered as classical. Let us finally mention that we shall keep our formalism Euclidean throughout our analysis. Transcription of our final results to Minkowski space-time will be made in the end. In this respect, characterizations such as 'Lorentz generators', 'Lorentz trace', etc. will be employed by abuse of language.

The quadratic part of the (pure) gauge field action reads

$$
S_{2}=\frac{1}{2} \alpha_{\mu}^{a}\left[-\left(D^{2}\right)^{a b} \delta_{\mu \nu}+\left(D_{\nu} D_{\mu}\right)^{a b}-\frac{1}{\xi}\left(D_{\mu} D_{\nu}\right)^{a b}-i g F_{\mu \nu}^{a b}\right] \alpha_{\nu}^{b}+\bar{c}^{a}\left[\left(D^{2}\right)^{a b}\right] c^{b},
$$

where $D_{\mu}^{a b}=D_{\mu}^{a b}(B)=\partial_{\mu} \delta^{a b}+g f^{a b c} B_{\mu}$ is the covariant field derivative in the adjoint representation, while $c, \bar{c}$ are the ghost fields. Obviously, $F_{\mu \nu}$ entering Eq. (2.1) is the

\footnotetext{
${ }^{2}$ For convenience we drop the characterization 'worldline' from hereon, even though we recognize the fact that we are using a term that has been established for the characterization of the same author's path integral pertaining to string quantization.
} 
Maxwell tensor for the background gauge field, i.e

$$
F_{\mu \nu}^{a b}=F_{\mu \nu}^{a b}(B)=-i f^{a b c} F_{\mu \nu}^{c}(B)=-i f^{a b c}\left(\partial_{\mu} B_{\nu}^{c}-\partial_{\nu} B_{\mu}^{c}-g f^{c d e} B_{\mu}^{d} B_{\nu}^{e}\right) .
$$

Adopting the Feynman gauge $(\xi=1)$ and introducing the Lorentz generators under which four-vectors transform, namely

$$
\left(J_{\rho \sigma}\right)_{\mu \nu}=i\left(\delta_{\rho \mu} \delta_{\sigma \nu}-\delta_{\rho \nu} \delta_{\sigma \mu}\right),
$$

we rewrite Eq. (2.1) as follows

$$
S_{2}=\frac{1}{2} \alpha_{\mu}^{a}\left[-\left(D^{2}\right)^{a b} \delta_{\mu \nu}-g(J \cdot F)_{\mu \nu}^{a b}\right] \alpha_{\nu}^{b}+\bar{c}^{a}\left[\left(D^{2}\right)^{a b}\right] c^{b} .
$$

In the one loop approximation, to which we shall restrict our considerations in this work, the effective action, as a functional of the background field, is given by

$$
\Gamma_{1}[B]=\frac{1}{2} \operatorname{Tr} \ln \left(-D^{2}-g J \cdot F\right)-\operatorname{Tr} \ln \left(-D^{2}\right)=\Gamma_{1, \text { gluons }}[B]+\Gamma_{\text {ghosts }}[B] .
$$

In what follows it suffices to work with $\Gamma_{1, \text { gluons }}[B]$ as $\Gamma_{\text {ghosts }}[B]$ is simply given by the first of the two terms entering the gluon contribution to the effective action, multiplied by $(-2)$.

Employing Schwinger's parametrization formula [22] we write (trace on 'Lorentz' and color indices)

$$
\Gamma_{1, \text { gluons }}[B]=-\frac{1}{2} \int_{0}^{\infty} \frac{d T}{T} \int d^{D} x \operatorname{Tr} K(x, x ; T)
$$

where

$$
K(y, x ; T)_{\mu \nu}^{a b} \equiv\left\langle y\left|e^{-T\left(-D^{2}-g J \cdot F\right)}\right| x\right\rangle_{\mu \nu}^{a b}
$$

corresponds to the (dynamical) gauge field propagator kernel in the background field.

The world-line path integral for $K(y, x ; T)_{\mu \nu}^{a b}$ results through standard procedures (see, e.g., Ref. [30]) and reads

$K(y, x ; T)_{\mu \nu}^{a b}=\int_{x(0)=x, x(T)=y} D x(t) \exp \left[-\frac{1}{4} \int_{0}^{T} d t \dot{x}^{2}(t)\right] P \exp \left[i g \int_{0}^{T} d t \dot{x} \cdot B+g \int_{0}^{T} d t J \cdot F\right]_{\mu \nu}^{a b}$.

As already established in Refs. [30,31] the Polyakov path integral results once we apply the 'area derivative' operator [33,34] given by

$$
\frac{\delta}{\delta s_{\mu \nu}(t)} \equiv \lim _{\varepsilon \rightarrow 0} \int_{-\varepsilon}^{\varepsilon} d s s \frac{\delta^{2}}{\delta x_{\mu}\left(t+\frac{s}{2}\right) \delta x_{\nu}\left(t-\frac{s}{2}\right)}
$$


and use, at the same time, the identities

$\frac{\delta}{\delta s_{\mu \nu}(t)} P \exp \left(i g \int_{0}^{T} d t \dot{x} \cdot B\right)^{a b}=\mathrm{P} \exp \left(i g \int_{t}^{T} d t \dot{x} \cdot B\right)_{\mu \rho}^{a a_{1}}(-i g F(x(t)))_{\rho \sigma}^{a_{1} a_{2}} \mathrm{P} \exp \left(i g \int_{0}^{t} d t \dot{x} \cdot B\right)_{\sigma \nu}^{a_{2} b}$

and

$$
\int_{0}^{T} d t \frac{\delta}{\delta s_{\mu \nu}(t)} \exp \left(-\frac{1}{4} \int_{0}^{T} d t \dot{x}^{2}\right)=\frac{1}{2} \int_{0}^{T} d t \omega_{\mu \nu}[\dot{x}(t)] \exp \left(-\frac{1}{4} \int_{0}^{T} d t \dot{x}^{2}\right)
$$

where $\omega_{\mu \nu}$ expresses the rotation of the vector tangent to the trajectory [26] and for paths described by differentiable functions assumes the form

$$
\omega_{\mu \nu}[\dot{x}]=\frac{T}{2}\left(\ddot{x}_{\mu} \dot{x}_{\nu}-\dot{x}_{\mu} \ddot{x}_{\nu}\right) .
$$

A more careful discussion pertaining to the spin factor is conducted in the appendix.

Once performing a partial integration, Eq. (2.8) assumes its Polyakov path-integral form which reads

$$
K(y, x ; T)_{\mu \nu}^{a b}=\int_{x(0)=x, x(T)=y} D x(t) \exp \left(-\frac{1}{4} \int_{0}^{T} d t \dot{x}^{2}\right) \mathrm{P} \exp \left(\frac{i}{2} \int_{0}^{T} d t J \cdot \omega\right)_{\mu \nu} \mathrm{P} \exp \left(i g \int_{0}^{T} d t \dot{x} \cdot B\right)^{a b} .
$$

In turn, the corresponding expression for the effective action, including the contribution from the ghost term, becomes

$$
\Gamma_{1}[B]=-\frac{1}{2} \int_{0}^{\infty} \frac{d T}{T} \int_{x(0)=x(T)} D x(t) \exp \left(-\frac{1}{4} \int_{0}^{T} d t \dot{x}^{2}\right)\left\{\operatorname{Tr}_{L} \Phi^{[1]}[\dot{x}]-2\right\} \operatorname{Tr}_{c} P \exp \left(i g \int_{0}^{T} d t \dot{x} \cdot B\right),
$$

where the indices on the traces stand for 'Lorentz' $(L)$ and 'color' $(c)$ and where

$$
\Phi^{[1]}[\dot{x}]_{\mu \nu} \equiv \mathrm{P} \exp \left[\frac{i}{2} \int_{0}^{T} d t J \cdot \omega[\dot{x}(t)]\right]_{\mu \nu}
$$

is the spin-factor expression? for the spin-1 particle-like entity (gluon) propagating in (Euclidean)space-time. It is not difficult to see that the spin-factor has a restricted

\footnotetext{
${ }^{3}$ Strictly speaking, Polyakov's spin-factor is given by the (Lorentz) trace of $\Phi^{[1]}[\dot{x}]_{\mu \nu}$.
} 
dependence on a path's profile. As argued in Ref. [30] and further deliberated on in the appendix, contributions of the spin-factor to the path integral comes solely from points where a four-momentum is applied through an emission or absorbtion of a gauge field quantum. Roughly speaking, this has to do with the fact that the expectation value $<\ddot{x}_{\mu} \dot{x}_{\nu}>-<\dot{x}_{\mu} \ddot{x}_{\nu}>$, as computed through the path integral, vanishes unless a four-momentum $k_{\mu}$ is imparted at the point $x$.

For the sake of comparison we give the corresponding expression for the one fermionic loop expression which contributes to the effective action [28]. It reads (color matrices in the fundamental representation)

$$
\Gamma_{1, \text { fermions }}[B]=\frac{1}{2} \int_{0}^{\infty} \frac{d T}{T} \int_{x(0)=x(T)} D x(t) \exp \left(-\frac{1}{4} \int_{0}^{T} d t \dot{x}^{2}\right) \operatorname{Tr}_{L} \Phi^{[1 / 2]}[\dot{x}] \operatorname{Tr}_{c} P \exp \left(i g \int_{0}^{T} d t \dot{x} \cdot B\right)
$$

with the spin factor now given by

$$
\Phi^{[1 / 2]}[\dot{x}] \equiv \mathrm{P} \exp \left[\frac{i}{2} \int_{0}^{T} d t S \cdot \omega[\dot{x}(t)]\right]
$$

and where the corresponding Lorentz generators belong to the spinor representation, i.e.

$$
S_{\mu \nu}=\frac{1}{2} \sigma_{\mu \nu}=\frac{i}{4}\left[\gamma_{\mu}, \gamma_{\nu}\right]
$$

Generally put, the Polyakov path integral recasting of a relativistic, quantum field theoretical system provides a unified basis for the description of the propagating particle-like entity; simply one has to adjust the weight provided by the spin-factor to its particular form. Thus, for a spin-zero particle the relevant weight factor is, simply, unity (note, in this regard, that ghosts fall into this class irrespective of the anticommutation relations, cf. minus sign, they obey) while for spin-1/2 and spin-1 particle-like entities the corresponding weights are provided by Eqs. (2.17) and (2.15), respectively.

For completeness let us mention that the path integral expression for the gluonic Green's function, namely

$$
i G(y, x)_{\mu \nu}^{a b}=\int_{0}^{\infty} d T K(y, x ; T)_{\mu \nu}^{a b}
$$

is determined once the substitution from Eq. (2.13) is made for the propagation kernel.

\section{The one Gluon Loop, M-point Effective Action}

In this Section we shall perform a number of manipulations through which we shall arrive to ready-to-apply master expressions for the computation of one-loop effective action 
terms. Let us commence our efforts by substituting plane wave solutions of the (classical) Yang-Mills equations satisfied by the background field, i.e. setting $B_{\mu}(x)=t_{G}^{a_{n}} \varepsilon_{\mu}^{n} e^{i p_{n} \cdot x}$ for each gauge field entering the M-th order term in the expansion of the Wilson exponential in (2.14). We obtain

$$
\begin{aligned}
& \Gamma_{1}^{(M)}\left(p_{1}, \ldots, p_{M}\right)=-\frac{1}{2}(i g)^{M} \operatorname{Tr}_{C}\left(t_{G}^{a_{M}} \ldots t_{G}^{a_{1}}\right) \int_{0}^{\infty} \frac{d T}{T}\left[\prod_{n=M}^{1} \int_{0}^{T} d t_{n}\right] \theta\left(t_{M}, \ldots, t_{1}\right) \\
& \times \int_{x(0)=x(T)} D x(t) \prod_{n=M}^{1} \varepsilon^{n} \cdot \dot{x}\left(t_{n}\right)\left\{\operatorname{Tr}_{L} \Phi^{[1]}[\dot{x}]-2\right\} \exp \left[-\frac{1}{4} \int_{0}^{T} d t \dot{x}^{2}+i \sum_{n=1}^{M} p_{n} \cdot x\left(t_{n}\right)\right]
\end{aligned}
$$

+permutations,

where $\theta\left(t_{M}, \ldots, t_{1}\right)=\prod_{n=M-1}^{1} \theta\left(t_{n+1}-t_{n}\right)$ and where the indication permutations refers to all possible rearrangements of the $t_{n}$ and the $t_{G}^{a_{n}}$ associated with the, $M$ in number, background gauge fields.

Our computational strategy for confronting the above quantity coincides with the one employed in Ref. [31]. It relies on a move to recast the spin-factor expression into an explicitly path-independent form. Once this is done the path integration can be immediately performed given that the 'action functional' is a simple gaussian (with a linear term). Subsequently, we shall deal with the spin-factor.

Following the procedure employed in the aformentioned reference we introduce the Grassmann variables $\bar{\xi}_{n}$ and $\xi_{n}$ through which the $\varepsilon^{n} \cdot \dot{x}\left(t_{n}\right)$ factors in Eq. (3.1) are elevated into exponentials according to [13]

$$
i \varepsilon^{n} \cdot \dot{x}\left(t_{n}\right)=\int d \xi_{n} d \bar{\xi}_{n} \exp \left[i \xi_{n} \bar{\xi}_{n} \varepsilon^{n} \cdot \dot{x}\left(t_{n}\right)\right]
$$

After substituting in (3.1) we obtain

$$
\begin{aligned}
& \Gamma_{1}^{(M)}\left(p_{1}, \ldots, p_{M}\right)=-\frac{1}{2} g^{M} \operatorname{Tr}_{C}\left(t_{G}^{a_{M}} \ldots t_{G}^{a_{1}}\right) \int_{0}^{\infty} \frac{d T}{T}\left[\prod_{n=M}^{1} \int_{0}^{T} d t_{n}\right] \theta\left(t_{M}, \ldots, t_{1}\right)\left[\prod_{n=M}^{1} \int d \xi_{n} d \bar{\xi}_{n}\right] \\
& \times \int_{x(0)=x(T)} D x(t)\left\{\operatorname{Tr}_{L} \Phi^{[1]}[\dot{x}]-2\right\} \exp \left[-\frac{1}{4} \int_{0}^{T} d t \dot{x}^{2}+i \sum_{n=1}^{M} \hat{k}\left(t_{n}\right) \cdot x\left(t_{n}\right)\right] \\
& + \text { permutations, }
\end{aligned}
$$

having set

$$
\hat{k}_{\mu}\left(t_{n}\right) \equiv p_{n, \mu}+\bar{\xi}_{n} \xi_{n} \varepsilon_{\mu}^{n} \frac{\partial}{\partial t_{n}} .
$$


Recalling the original specification of the spin-factor, which utilizes the employment of the area derivative, let us rewrite Eq. (3.3) in the specific form which takes into account the fact that the gauge potentials entering the expansion of the Wilson exponential are plane waves. For the $M t h$ order term we write

$$
\begin{aligned}
\operatorname{Tr}_{L} \Phi^{[1]}[\hat{k}] \equiv & \exp \left[-i \sum_{n=1}^{M} \hat{k}\left(t_{n}\right) \cdot x\left(t_{n}\right)\right]\left[\operatorname{Tr}_{L} \mathrm{P} \exp \left(-i \int_{0}^{T} d t J \cdot \frac{\delta}{\delta s}\right)\right] \\
& \times \exp \left[i \sum_{n=1}^{M} \hat{k}\left(t_{n}\right) \cdot x\left(t_{n}\right)\right] .
\end{aligned}
$$

The above expression illustrates in an immediate, albeit formal, manner the pathindependence of the spin-factor: One observes that the area derivative acting on the exponential will produce delta functions entering the parametric integration, cf. Eq. (2.9), entering the definition of the area derivative operator. A well defined argument leading to this result is provided in the appendix.

Returning to the case in hand, we write

$$
\begin{aligned}
& \Gamma_{1}^{(M)}\left(p_{1}, . ., p_{M}\right)=-\frac{1}{2} g^{M} \operatorname{Tr}_{C}\left(t_{G}^{a_{M}} \ldots t_{G}^{a_{1}}\right) \int_{0}^{\infty} \frac{d T}{T}\left[\prod_{n=M}^{1} \int_{0}^{T} d t_{n}\right] \theta\left(t_{M}, \ldots, t_{1}\right)\left[\prod_{n=M}^{1} \int d \xi_{n} d \bar{\xi}_{n}\right] \\
& \times\left\{\operatorname{Tr}_{L} \Phi^{[1]}[\hat{k}]-2\right\} \int_{x(0)=x(T)} D x(t) \exp \left[-\frac{1}{4} \int_{0}^{T} d t \dot{x}^{2}+i \sum_{n=1}^{M} \hat{k}\left(t_{n}\right) \cdot x\left(t_{n}\right)\right] \\
& + \text { permutations. }
\end{aligned}
$$

The first task we shall carry out is to perform the, basically Gaussian, path integral. Straightforward manipulations, partly displayed in the appendix, lead to the result

$$
\begin{aligned}
& \quad \int_{x(0)=x(T)} D x(t) \exp \left[-\frac{1}{4} \int_{0}^{T} d t \dot{x}^{2}(t)+i \sum_{n=1}^{M} \hat{k}\left(t_{n}\right) \cdot x\left(t_{n}\right)\right]=(2 \pi)^{D} \delta^{(D)}\left(\sum_{n=1}^{M} p_{n}\right) \frac{1}{(4 \pi T)^{D / 2}} \\
& \times \exp \left[\sum_{n<m} p_{n} \cdot p_{m} G\left(t_{n}, t_{m}\right)+\sum_{n \neq m} \bar{\xi}_{n} \xi_{n} \varepsilon^{n} \cdot p_{m} \partial_{n} G\left(t_{n}, t_{m}\right)\right. \\
& \left.+\frac{1}{2} \sum_{n \neq m} \bar{\xi}_{n} \xi_{n} \bar{\xi}_{m} \xi_{m} \varepsilon^{n} \cdot \varepsilon^{m} \partial_{n} \partial_{m} G\left(t_{n}, t_{m}\right)\right] .
\end{aligned}
$$

One notes contributions pertaining solely to the points of attachment of external gauge field on the loop contour. In the above expression the following Green's function [13] has been employed

$$
G\left(t, t^{\prime}\right)=\left|t-t^{\prime}\right|\left[1-\frac{\left|t-t^{\prime}\right|}{T}\right]
$$


It corresponds to the motion of a one-dimensional particle moving on a closed contour, i.e.

$$
\frac{1}{2} \frac{\partial^{2}}{\partial t^{2}} G\left(t, t^{\prime}\right)=-\delta\left(t-t^{\prime}\right)+\frac{1}{T}
$$

and obeys the boundary conditions $G\left(0, t^{\prime}\right)=G\left(T, t^{\prime}\right)$ and $\dot{G}\left(0, t^{\prime}\right)=\dot{G}\left(T, t^{\prime}\right)$.

Introducing the dimensionless parameters $u_{i}$ according to $t_{i}=T u_{i}, i=1, \ldots, n$, the interim result for $\Gamma_{1}^{(M)}\left(p_{1}, \ldots, p_{M}\right)$ reads

$$
\begin{aligned}
& \Gamma_{1}^{(M)}\left(p_{1}, \ldots, p_{M}\right)=-\frac{1}{2} g^{M}(2 \pi)^{D} \delta^{(D)}\left(\sum_{n=1}^{M} p_{n}\right) \operatorname{Tr}_{C}\left(t_{G}^{a_{M}} \cdots t_{G}^{a_{1}}\right) \frac{1}{(4 \pi)^{D / 2}} \int_{0}^{\infty} d T T^{M-D / 2-1} \\
& \times\left[\prod_{n=M}^{1} \int_{0}^{1} d u_{n}\right] \theta\left(u_{M}, \ldots, u_{1}\right) F^{(M)}\left(u_{1}, \ldots, u_{M} ; T\right) \exp \left[T \sum_{n<m} p_{n} \cdot p_{m} G\left(u_{n}, u_{m}\right)\right] \\
& \quad+\text { permutations, }
\end{aligned}
$$

where $G\left(u_{n}, u_{m}\right)=\left|u_{n}-u_{m}\right|\left[1-\left|u_{n}-u_{m}\right|\right]$ satisfies the additional properties

$$
\partial_{n} G\left(u_{n}, u_{m}\right) \equiv \dot{G}\left(u_{n}, u_{m}\right)=\operatorname{sign}\left(u_{n}-u_{m}\right)-2\left(u_{n}-u_{m}\right)=-\dot{G}\left(u_{m}, u_{n}\right)
$$

and

$$
-\partial_{n} \partial_{m} G\left(u_{n}, u_{m}\right)=\partial_{n}^{2} G\left(u_{n}, u_{m}\right) \equiv \ddot{G}\left(u_{n}, u_{m}\right)=2\left[\delta\left(u_{n}-u_{m}\right)-1\right] .
$$

Finally, in Eq. (3.10) we have set

$$
\begin{aligned}
& F^{(M)}\left(u_{1}, \ldots, u_{M} ; T\right)=\left[\prod_{n=M}^{1} \int d \xi_{n} d \bar{\xi}_{n}\right]\left(\operatorname{Tr}_{L} \Phi^{[1]}[\hat{k}]-2\right) \\
& \times \exp \left[\sum_{n \neq m} \bar{\xi}_{n} \xi_{n} \varepsilon^{n} \cdot p_{m} \partial_{n} G\left(u_{n}, u_{m}\right)\right. \\
& \left.+\frac{1}{2 T} \sum_{n \neq m} \bar{\xi}_{n} \xi_{n} \bar{\xi}_{m} \xi_{m} \varepsilon^{n} \cdot \varepsilon^{m} \partial_{n} \partial_{m} G\left(u_{n}, u_{m}\right)\right] .
\end{aligned}
$$

The spin-factor can now be brought into a ready-to-apply form through a series of manipulations that are outlined in the appendix. The following result is arrived at

$$
\begin{gathered}
\Phi_{\mu \nu}^{[1]}[\hat{k}]=\mathrm{P} \exp \left[\frac{i}{2} \sum_{n=1}^{M} J \cdot \phi(n)\right]_{\mu \nu}=\delta_{\mu \nu}+\frac{i}{2}\left(J_{\rho \sigma}\right)_{\mu \nu} \sum_{n=1}^{M} \phi_{\rho \sigma}(n) \\
+\left(\frac{i}{2}\right)^{2}\left(J_{\rho_{2} \sigma_{2}}\right)_{\mu \lambda}\left(J_{\rho_{1} \sigma_{1}}\right)_{\lambda \nu} \sum_{n_{2}=1}^{M} \sum_{n_{1}=1}^{n_{2}-1} \phi_{\rho_{2} \sigma_{2}}\left(n_{2}\right) \phi_{\rho_{1} \sigma_{1}}\left(n_{1}\right)+\ldots
\end{gathered}
$$

\footnotetext{
${ }^{4}$ To be exact, the solution of Eq. (3.9) includes an arbitrary constant which does not appear in Eq. (3.8) on the account that momentum conservation has been imposed.
} 
with

$$
\phi_{\mu \nu}(n)=2 \bar{\xi}_{n} \xi_{n}\left(\varepsilon_{\mu}^{n} p_{n, \nu}-\varepsilon_{\nu}^{n} p_{n, \mu}\right)+\frac{4}{T} \bar{\xi}_{n+1} \xi_{n+1} \bar{\xi}_{n} \xi_{n}\left(\varepsilon_{\mu}^{n+1} \varepsilon_{\nu}^{n}-\varepsilon_{\nu}^{n+1} \varepsilon_{\mu}^{n}\right) \delta\left(u_{n+1}-u_{n}\right)
$$

and where we have designated that $\bar{\xi}_{M+1}=\xi_{M+1}=0$.

Two observations of practical interest can be made in connection with the above expression for the spin-factor. First, it is clear that the number of terms in the expansion of the exponential in Eq. (3.14) terminates at $M$ as the saturation point of the Grassman variables is by then reached. Second, the delta function containing term in (3.14) implies that for a given ordering there is a contribution from coinciding points, $u_{n}$ and $u_{n+1}$ in this case. This occurrence signifies the presence of a 'four-gluon vertex' which is automatically included in a given perturbative calculation, along with the (derivativedependent) 'three-gluon vertices' represented by the first term. One thereby concludes that the $M t h$ order perturbative contributions to the effective action are classified, via the spin-factor, exclusively by the number of the points of gluon (single or pairwise) attachements on the closed world-line contour, in all possible permutations. Accordingly, the computation of the M-point effective action term will collect all $M$ th order, 1PI Feynman diagrams.

We mention in passing that fermionic loop contributions to the effective action easily follow by referring to Eqs. (2.16)-(2.18). One simply has to make the substitution $\phi_{\rho \sigma}\left(J_{\rho \sigma}\right)_{\mu \nu} \rightarrow S_{\mu \nu} \phi_{\mu \nu}$ in Eqs. (3.14) and (3.15) and use the fundamental representation of the group.

Given the expressions we have arrived at, what remains to be carried out are the integrations over the Grassmann variables as well as the parametric integrations entering Eq. (3.10). Numerical methods have been developed for this purpose whose report is forthcoming [32]. For the rest of this paper we restrict ourselves to the computation of the divergent part of the effective action. In this regard, let us observe, by looking at Eq. (3.13), that ultraviolet divergencies will occur only for terms of order $M=$ $2,3,4$. Specifically, by focusing on the terms that have the minimum number of $p_{n, \mu}$ factors one determines, through dimensional considerations, that they should carry the compensating factor $T^{2-M}$ for $M=2,3,4$. The latter, combines with $T^{M-1-D / 2}$ in (3.10) to produce divergent terms $\sim \Gamma\left(2-\frac{D}{2}\right)$. Further inspection shows that no such terms arise for $M \geq 5$, a fact that directly complies with the renormalizability of the theory.

As our final remark in this Section, let us mention that our manipulations up to now have treated the gluons as 'real', i.e. we have assumed that

$$
\varepsilon^{n} \cdot p_{n}=0 \quad \text { and } \quad p_{n}^{2}=0, \quad n=1,2, \cdots
$$

In the following Section, where our computations will run against infra-red problems, we shall protect our expressions from corresponding divergencies by going slightly off mass shell. i.e. by setting $p_{n}^{2}=\lambda^{2}$, with $\lambda^{2}>\Lambda_{Q C D}^{2}$. 


\section{Computation of Divergent one-loop Effective Ac- tion Terms to Fourth Order}

In this Section we shall apply our comprehesive formulas given by Eqs. (3.10) and (3.13)(3.15) towards the computation of the divergent contributions to the $M=2,3,4$ terms in the expansion of the effective action -in fact, the only terms which exhibit ultra-violet divergencies. We leave the task of computing finite contributions, to the same order, to a future paper where numerical methods will be applied.

\subsection{The two gluon contribution $(M=2)$ to the Effective Action}

The present calculation pertains to the one gluon loop configuration with two (truncated) gluon and ghost attachments. Our master expression accomodates all the contributing Feynman diagrams. From Eqs. (3.14) and (3.15) we determine, for $M=2$,

$$
\operatorname{Tr}_{L} \Phi^{[1]}=D-8 \bar{\xi}_{1} \xi_{1} \bar{\xi}_{2} \xi_{2} \varepsilon^{1} \cdot \varepsilon^{2} p_{1} \cdot p_{2}
$$

Upon substituting in (3.13) and performing the Grassmann integrations we obtain

$$
F^{(M=2)}\left(u_{1}, u_{2} ; T\right)=-\frac{1}{T}(D-2) \varepsilon^{1} \cdot \varepsilon^{2} \ddot{G}\left(u_{1}, u_{2}\right)-8 \varepsilon^{1} \cdot \varepsilon^{2} p_{1} \cdot p_{2} .
$$

One notes that the delta function entering the specification of $\ddot{G}\left(u_{1}, u_{2}\right)$ accomodates the contribution coming from the class of Feynman diagrams wherein the two (truncated) external gluons attach themselves to the loop through a four-point vertex.

The above result when substituted in (3.13) gives, after an integration by parts which results in the replacement $\ddot{G}\left(u_{1}, u_{2}\right) \rightarrow-T p_{1} \cdot p_{2} \dot{G}^{2}\left(u_{1}, u_{2}\right)$,

$$
\begin{aligned}
& \Gamma_{1}^{(M=2)}\left(p_{1}, p_{2}\right)=-\frac{1}{2}(2 \pi)^{4} \delta^{(4)}\left(p_{1}+p_{2}\right) \operatorname{Tr}_{C}\left(t_{G}^{a_{2}} t_{G}^{a_{1}}\right) \frac{g^{2}}{(4 \pi)^{D / 2}} \varepsilon^{1} \cdot \varepsilon^{2} p_{1} \cdot p_{2} \\
& \times \int_{0}^{\infty} d T T^{1-D / 2} \int_{0}^{1} d u_{2} \int_{0}^{u_{2}} d u_{1}\left[(D-2) \dot{G}^{2}\left(u_{1}, u_{2}\right)-8\right] \exp \left[-T \lambda^{2} G\left(u_{1}, u_{2}\right)\right] \\
& + \text { permutations, }
\end{aligned}
$$

where the infra-red cutoff $\lambda$ has been introduced by going off shell. The integrations in the last equation can be easily performed and lead to the final result

$$
\begin{aligned}
\Gamma_{1}^{(M=2)}\left(p_{1}, p_{2}\right)= & -\frac{1}{2}(2 \pi)^{4} \delta^{(4)}\left(p_{1}+p_{2}\right) N \delta^{a_{2} a_{1}} \frac{g^{2}}{(4 \pi)^{2}}\left(4 \pi \frac{\mu^{2}}{\lambda^{2}}\right)^{2-D / 2} \varepsilon^{1} \cdot \varepsilon^{2} p_{1} \cdot p_{2} \\
& \times \Gamma\left(2-\frac{D}{2}\right) \frac{11-7(2-D / 2)}{3-2(2-D / 2)} B\left(\frac{D}{2}-1, \frac{D}{2}-1\right),
\end{aligned}
$$

where the adjustment $g^{2} \rightarrow g_{D}^{2}=g^{2} \mu^{4-D}$ was made in order to restore dimensional consistency. The term permutations in Eq. (3.10) has been duly taken care of by taking 
into account all the rearrangements of indices $(1,2)$ and dividing by 2 ! in order to comply with boson non-distinguishability.

From Eq. (4.4) we verify, once returning to Minkowski space-time, the well known result (which does not take into account the contribution from the fermionic loop)

$$
Z_{g}=1-\frac{1}{2} \frac{g^{2}}{(4 \pi)^{2}} N \frac{11}{3} \frac{1}{2-D / 2}
$$

It is of interest to observe, by referring to Eqs. (2.13), (2.19) and as has been explicitly demonstrated in Refs. [30,31], that the corresponding formulas resulting from Polyakov's path-integral for open lines have the same basic structure with the ones that have resulted from the present considerations pertaining to loops. It, then, becomes a straight forward matter to surmise the validity of Ward's identity $Z_{B}^{1 / 2} Z_{g}=1$ which is known to hold in the framework of the background gauge fixing method.

\subsection{The three gluon contribution $(\mathrm{M}=3)$ to the Effective Action}

We now turn our attention to $\Gamma_{1}^{(M=3)}$ which summarizes the contributions from the classes of Feynman diagrams involving three (truncated) external guons, as well as ghosts. Again, our first task is to compute the corresponding expression for the spin-factor. Eqs. (3.14) and (3.15) now give

$$
\begin{aligned}
\operatorname{Tr}_{L} \Phi^{[1]}= & D+8 \bar{\xi}_{2} \xi_{2} \bar{\xi}_{3} \xi_{3}\left(\varepsilon^{2} \cdot p_{3} \varepsilon^{3} \cdot p_{2}-\varepsilon^{2} \cdot \varepsilon^{3} p_{2} \cdot p_{3}\right)+8 \bar{\xi}_{1} \xi_{1} \bar{\xi}_{3} \xi_{3}\left(\varepsilon^{1} \cdot p_{3} \varepsilon^{3} \cdot p_{1}-\varepsilon^{1} \cdot \varepsilon^{3} p_{1} \cdot p_{3}\right) \\
& +8 \bar{\xi}_{1} \xi_{1} \bar{\xi}_{2} \xi_{2}\left(\varepsilon^{1} \cdot p_{2} \varepsilon^{2} \cdot p_{1}-\varepsilon^{1} \cdot \varepsilon^{2} p_{1} \cdot p_{2}\right) \\
& +\frac{16}{T} \bar{\xi}_{1} \xi_{1} \bar{\xi}_{2} \xi_{2} \bar{\xi}_{3} \xi_{3}\left[\left(\varepsilon^{1} \cdot \varepsilon^{2} \varepsilon^{3} \cdot p_{1}-\varepsilon^{1} \cdot \varepsilon^{3} \varepsilon^{2} \cdot p_{1}\right) \delta\left(u_{3}-u_{2}\right)\right. \\
& \left.+\left(\varepsilon^{1} \cdot \varepsilon^{3} \varepsilon^{2} \cdot p_{3}-\varepsilon^{2} \cdot \varepsilon^{3} \varepsilon^{1} \cdot p_{3}\right) \delta\left(u_{2}-u_{1}\right)+T \varepsilon^{1} \cdot p_{3} \varepsilon^{2} \cdot p_{1} \varepsilon^{3} \cdot p_{2}\right]
\end{aligned}
$$

The integration over the Grassmann variables can be systematically performed, yielding the result

$$
\begin{aligned}
& F_{1}^{(M=3)}\left(u_{1}, u_{2}, u_{3} ; T\right)=-\frac{D-2}{T}\left\{\varepsilon^{1} \cdot \varepsilon^{2}\left[\varepsilon^{3} \cdot p_{1} \dot{G}\left(u_{3}, u_{1}\right)+\varepsilon^{3} \cdot p_{2} \dot{G}\left(u_{3}, u_{2}\right)\right] \ddot{G}\left(u_{1}, u_{2}\right)+\right. \\
& +\varepsilon^{1} \cdot \varepsilon^{3}\left[\varepsilon^{2} \cdot p_{1} \dot{G}\left(u_{2}, u_{1}\right)+\varepsilon^{2} \cdot p_{3} \dot{G}\left(u_{2}, u_{3}\right)\right] \ddot{G}\left(u_{1}, u_{3}\right)+\varepsilon^{2} \cdot \varepsilon^{3}\left[\varepsilon^{1} \cdot p_{2} \dot{G}\left(u_{1}, u_{2}\right)+\varepsilon^{1} \cdot p_{3} \dot{G}\left(u_{1}, u_{3}\right)\right] \\
& \left.\times \ddot{G}\left(u_{2}, u_{3}\right)\right\}+\frac{16}{T}\left(\varepsilon^{1} \cdot \varepsilon^{2} \varepsilon^{3} \cdot p_{1}-\varepsilon^{1} \cdot \varepsilon^{3} \varepsilon^{2} \cdot p_{1}\right) \delta\left(u_{3}-u_{2}\right) \\
& +\frac{16}{T}\left(\varepsilon^{1} \cdot \varepsilon^{3} \varepsilon^{2} \cdot p_{3}-\varepsilon^{2} \cdot \varepsilon^{3} \varepsilon^{1} \cdot p_{3}\right) \delta\left(u_{2}-u_{1}\right)+f . t .
\end{aligned}
$$

where f.t. stands for 'terms with finite contribution'. Obviously the latter involve terms with $T$ to the 0 th power or higher, equivalently, they involve more than one (external) momentum variables. Let us reiterate that the finite terms should be computable through numerical methods that are currently being developed. 
Substituting the above result in Eq. (3.10) we obtain

$$
\begin{aligned}
& \Gamma_{1}^{(M=3)}\left(p_{1}, p_{2}, p_{3}\right)=-\frac{1}{2}(2 \pi)^{4} \delta^{(4)}\left(p_{1}+p_{2}+p_{3}\right) \operatorname{Tr}_{C}\left(t_{G}^{a_{3}} t_{G}^{a_{2}} t_{G}^{a_{1}}\right) \frac{g^{3}}{(4 \pi)^{D / 2}} \int_{0}^{\infty} d T T^{1-D / 2} \\
& \int_{0}^{1} d u_{3} \int_{0}^{u_{3}} d u_{2} \int_{0}^{u_{2}} d u_{1} \times\left\{4(D-2)\left[\varepsilon^{1} \cdot \varepsilon^{2} \varepsilon^{3} \cdot p_{2}\left(u_{2}-u_{1}\right)+\varepsilon^{1} \cdot \varepsilon^{3} \varepsilon^{2} \cdot p_{1}\left(1-\left(u_{3}-u_{1}\right)\right)\right]\right. \\
& \left.+\varepsilon^{2} \cdot \varepsilon^{3} \varepsilon^{1} \cdot p_{3}\left(u_{3}-u_{2}\right)\right]-16\left(\varepsilon^{1} \cdot \varepsilon^{2} \varepsilon^{3} \cdot p_{2}+\varepsilon^{1} \cdot \varepsilon^{3} \varepsilon^{2} \cdot p_{1}\right) \delta\left(u_{3}-u_{2}\right) \\
& \left.-16\left(\varepsilon^{1} \cdot \varepsilon^{3} \varepsilon^{2} \cdot p_{1}+\varepsilon^{2} \cdot \varepsilon^{3} \varepsilon^{1} \cdot p_{3}\right) \delta\left(u_{2}-u_{1}\right)+f . t .\right\} \\
& \times \exp \left\{-\frac{T \lambda^{2}}{2}\left[\left(u_{2}-u_{1}\right)\left(1-\left(u_{2}-u_{1}\right)\right)+\left(u_{3}-u_{2}\right)\left(1-\left(u_{3}-u_{2}\right)\right)\right.\right. \\
& \left.\left.+\left(u_{3}-u_{1}\right)\left(1-\left(u_{3}-u_{1}\right)\right)\right]\right\}+ \text { permutations. }
\end{aligned}
$$

It is easy to see that the first term in the curly brackets takes care of the Feynman diagrams involving three-gluon vertices while the other two, which carry the delta-functions, collect the contributions from diagrams with one four-vertex. To further guide the reader let us also mention that use was made of Eqs. (3.11) and (3.12). Accordingly, the above expression refers to the specific ordering which enters these equations and underlies the particular integrations over the parameters $u_{1}, u_{2}$ and $u_{3}$, an occurrence that will be rectified shortly. Finally, in the exponential factor we have set

$$
p_{1}^{2}=p_{2}^{2}=p_{3}^{2}=\lambda^{2}, \quad 2 p_{1} \cdot p_{2}=2 p_{1} \cdot p_{3}=2 p_{2} \cdot p_{3}=-\lambda^{2} .
$$

Next, we make the variable change $u_{2}-u_{1}=x_{2}$ and $u_{3}-u_{1}=x_{3}$ which casts Eq. (4.8) into the form

$$
\begin{aligned}
& \Gamma_{1}^{(M=3)}\left(p_{1}, p_{2}, p_{3}\right)=-\frac{1}{2}(2 \pi)^{4} \delta^{(4)}\left(p_{1}+p_{2}+p_{3}\right) \operatorname{Tr}_{C}\left(t_{G}^{a_{3}} t_{G}^{a_{2}} t_{G}^{a_{1}}\right) g \frac{g^{2}}{(4 \pi)^{2}}\left(4 \pi \frac{\mu^{2}}{\lambda^{2}}\right)^{2-D / 2} \\
& \times\left\{-4(D-2) a_{D}\left(\varepsilon^{1} \cdot \varepsilon^{2} \varepsilon^{3} \cdot p_{2}+\varepsilon^{1} \cdot \varepsilon^{3} \varepsilon^{2} \cdot p_{1}+\varepsilon^{2} \cdot \varepsilon^{3} \varepsilon^{1} \cdot p_{3}\right)+8 b_{D}\left(\varepsilon^{1} \cdot \varepsilon^{2} \varepsilon^{3} \cdot p_{2}\right.\right. \\
& \left.\left.+3 \varepsilon^{1} \cdot \varepsilon^{3} \varepsilon^{2} \cdot p_{1}+2 \varepsilon^{2} \cdot \varepsilon^{3} \varepsilon^{1} \cdot p_{3}\right)\right\} \Gamma\left(2-\frac{D}{2}\right)+\text { f.t }+ \text { permutations }
\end{aligned}
$$

where we have introduced

$$
a_{D}=2^{2-D / 2} \int_{0}^{1} d x_{3} \int_{0}^{x_{3}} d x_{2} x_{2}\left[x_{2}\left(1-x_{2}\right)+\left(x_{3}-x_{2}\right)\left(1-x_{3}+x_{2}\right)+x_{3}\left(1-x_{3}\right)\right]^{D / 2-2}
$$

and

$$
b_{D}=\int_{0}^{1} d x_{3}\left(x_{3}\left(1-x_{3}\right)\right)^{D / 2-2} .
$$


Obviously $a_{4}=\frac{1}{6}$ and $b_{4}=1$.

In order to obtain the final result we need to take into account contributions coming from all permutations of the variables $u_{1}, u_{2}, u_{3}$ and divide by 3 ! to compensate for boson indistinguishability. The result can be easily obtained using the cyclic properties of the trace. One, finally, obtains

$$
\begin{aligned}
& \Gamma_{1}^{(M=3)}\left(p_{1}, p_{2}, p_{3}\right)=\frac{1}{2}(2 \pi)^{4} \delta^{(4)}\left(p_{1}+p_{2}+p_{3}\right) \operatorname{Tr}_{C}\left(t_{G}^{a_{3}} t_{G}^{a_{2}} t_{G}^{a_{1}}\right) g \frac{g^{2}}{(4 \pi)^{2}}\left(4 \pi \frac{\mu^{2}}{\lambda^{2}}\right)^{2-D / 2} \\
& \times\left\{4(D-2) a_{D}\left(\varepsilon^{1} \cdot \varepsilon^{2} \varepsilon^{3} \cdot p_{2}+\varepsilon^{1} \cdot \varepsilon^{3} \varepsilon^{2} \cdot p_{1}+\varepsilon^{2} \cdot \varepsilon^{3} \varepsilon^{1} \cdot p_{3}\right)-16 b_{D}\left(\varepsilon^{1} \cdot \varepsilon^{2} \varepsilon^{3} \cdot p_{2}\right.\right. \\
& \left.\left.+\varepsilon^{1} \cdot \varepsilon^{3} \varepsilon^{2} \cdot p_{1}+\varepsilon^{2} \cdot \varepsilon^{3} \varepsilon^{1} \cdot p_{3}\right)\right\} \Gamma\left(2-\frac{D}{2}\right)+\text { f.t. }
\end{aligned}
$$

in agreement with the known result.

\subsection{The four gluon contribution $(M=4)$ to the Effective Action}

The computation in the present subsection pertains to a 'world-line' diagram which collectively accomodates all the classes of the contributing Feynman diagrams, i.e. with zero, one and two four-vertices (plus, of course, contributions from ghost diagrams). As our present analytic computations refer to the divergent part, let us isolate the relevant contribution (terms with the factor $1 / T^{2}$ ) entering the expression for the spin-factor. We find

$\operatorname{Tr}_{L} \Phi^{[1]}=D+\frac{32}{T^{2}} \bar{\xi}_{4} \xi_{4} \bar{\xi}_{3} \xi_{3} \bar{\xi}_{2} \xi_{2} \bar{\xi}_{1} \xi_{1}\left(\varepsilon^{1} \cdot \varepsilon^{4} \varepsilon^{2} \cdot \varepsilon^{3}-\varepsilon^{1} \cdot \varepsilon^{3} \varepsilon^{2} \cdot \varepsilon^{4}\right) \delta\left(u_{4}-u_{3}\right) \delta\left(u_{2}-u_{1}\right)+f . t$.

Integration over the Grassmann variables is a straightforward matter and gives

$$
\begin{aligned}
& F^{(M=4)}\left(u_{4}, u_{3}, u_{2}, u_{1} ; T\right)=\frac{D-2}{T^{2}}\left[\varepsilon^{1} \cdot \varepsilon^{2} \varepsilon^{3} \cdot \varepsilon^{4} \ddot{G}\left(u_{1}, u_{2}\right) \ddot{G}\left(u_{3}, u_{4}\right)\right. \\
& \left.\left.+\varepsilon^{1} \cdot \varepsilon^{3} \varepsilon^{2} \cdot \varepsilon^{4} \ddot{G}\left(u_{1}, u_{3}\right) \ddot{G}\left(u_{2}, u_{4}\right)++\varepsilon^{1} \cdot \varepsilon^{4} \varepsilon^{2} \cdot \varepsilon^{3}\right) \ddot{G}\left(u_{1}, u_{4}\right) \ddot{G}\left(u_{2}, u_{3}\right)\right] \\
& +\frac{32}{T^{2}}\left(\varepsilon^{1} \cdot \varepsilon^{4} \varepsilon^{2} \cdot \varepsilon^{3}-\varepsilon^{1} \cdot \varepsilon^{3} \varepsilon^{2} \cdot \varepsilon^{4}\right) \delta\left(u_{4}-u_{3}\right) \delta\left(u_{2}-u_{1}\right)+f . t .
\end{aligned}
$$

Substituting the above expression into Eq. (3.10) we get

$$
\begin{aligned}
& \Gamma_{1}^{(M=4)}\left(p_{1}, p_{2}, p_{3}, p_{4}\right)=-\frac{1}{2}(2 \pi)^{4} \delta^{(4)}\left(p_{1}+p_{2}+p_{3}+p_{4}\right) \operatorname{Tr}_{C}\left(t_{G}^{a_{4}} t_{G}^{a_{3}} t_{G}^{a_{2}} t_{G}^{a_{1}}\right) \frac{g_{D}^{4}}{(2 \pi)^{D / 2}} \\
& \times \int_{0}^{1} d u_{4} \int_{0}^{u_{4}} d u_{3} \int_{0}^{u_{3}} d u_{2} \int_{0}^{u_{2}} d u_{1}\left\{4(D-2)\left(\varepsilon^{1} \cdot \varepsilon^{2} \varepsilon^{3} \cdot \varepsilon^{4}+\varepsilon^{1} \cdot \varepsilon^{3} \varepsilon^{2} \cdot \varepsilon^{4}+\varepsilon^{1} \cdot \varepsilon^{4} \varepsilon^{2} \cdot \varepsilon^{3}\right)\right. \\
& -4(D-2) \varepsilon^{1} \cdot \varepsilon^{2} \varepsilon^{3} \cdot \varepsilon^{4}\left[\delta\left(u_{2}-u_{1}\right)+\delta\left(u_{4}-u_{3}\right)\right]-4(D-2) \varepsilon^{1} \cdot \varepsilon^{4} \varepsilon^{2} \cdot \varepsilon^{3} \delta\left(u_{3}-u_{2}\right)+ \\
& +4(D-2) \varepsilon^{1} \cdot \varepsilon^{2} \varepsilon^{3} \cdot \varepsilon^{4} \delta\left(u_{2}-u_{1}\right) \delta\left(u_{4}-u_{3}\right)+32\left(\varepsilon^{1} \cdot \varepsilon^{4} \varepsilon^{2} \cdot \varepsilon^{3}-\varepsilon^{1} \cdot \varepsilon^{3} \varepsilon^{2} \cdot \varepsilon^{4}\right) \\
& \left.\times \delta\left(u_{2}-u_{1}\right) \delta\left(u_{4}-u_{3}\right)\right\}\left[\sum_{n=1}^{4} \sum_{m=n+1}^{4} p_{n} \cdot p_{m} G\left(u_{n}, u_{m}\right)\right]^{D / 2-2} \Gamma\left(2-\frac{D}{2}\right) \\
& + \text { f.t. }+ \text { permutations. }
\end{aligned}
$$


One can easily verify that the first term inside the curly brackets represents contributions corresponding to the Feynman diagrams with no four-vertices, the next two to those with one and the last to those with two. Of course, the above expression pertains to a particular ordering of the variables $u_{1}, u_{2}, u_{3}, u_{4}$ as reflected in the explicit delta functions which make their entrance.

Performing the parametric integrations, in the specific ordering that appears in Eq. (4.16), one obtains

$$
\begin{aligned}
& \Gamma_{1}^{(M=4)}\left(p_{1}, p_{2}, p_{3}, p_{4}\right)=-\frac{1}{2}(2 \pi)^{4} \delta^{(4)}\left(p_{1}+p_{2}+p_{3}+p_{4}\right) \operatorname{Tr}_{C}\left(t_{G}^{a_{4}} t_{G}^{a_{3}} t_{G}^{a_{2}} t_{G}^{a_{1}}\right) \frac{g_{D}^{4}}{(2 \pi)^{D / 2}} \\
& \times\left\{4(D-2) A_{D}\left(\varepsilon^{1} \cdot \varepsilon^{2} \varepsilon^{3} \cdot \varepsilon^{4}+\varepsilon^{1} \cdot \varepsilon^{3} \varepsilon^{2} \cdot \varepsilon^{4}+\varepsilon^{1} \cdot \varepsilon^{4} \varepsilon^{2} \cdot \varepsilon^{3}\right)-4(D-2)\left(B_{D}-C_{D}\right) \varepsilon^{1} \cdot \varepsilon^{2} \varepsilon^{3} \cdot \varepsilon^{4}-\right. \\
& \left.-4(D-2) D_{D} \varepsilon^{1} \cdot \varepsilon^{4} \varepsilon^{2} \cdot \varepsilon^{3}+32 C_{D}\left(\varepsilon^{1} \cdot \varepsilon^{4} \varepsilon^{2} \cdot \varepsilon^{3}-\varepsilon^{1} \cdot \varepsilon^{3} \varepsilon^{2} \cdot \varepsilon^{4}\right)\right\} \Gamma\left(2-\frac{D}{2}\right)+f . t \\
& \quad+\text { permutations, }
\end{aligned}
$$

where we have set

$$
\begin{aligned}
A_{D} & \equiv \int_{0}^{1} d u_{4} \int_{0}^{u_{4}} d u_{3} \int_{0}^{u_{3}} d u_{2} \int_{0}^{u_{2}} d u_{1}\left[\sum_{n=1}^{4} \sum_{m=n+1}^{4} p_{n} \cdot p_{m} G\left(u_{n}, u_{m}\right)\right]^{D / 2-2} \\
B_{D} & \equiv \int_{0}^{1} d u_{4} \int_{0}^{u_{4}} d u_{3} \int_{0}^{u_{3}} d u_{2} \int_{0}^{u_{2}} d u_{1}\left[\delta\left(u_{2}-u_{1}\right)+\delta\left(u_{4}-u_{3}\right)\right]\left[\sum_{n=1}^{4} \sum_{m=n+1}^{4} p_{n} \cdot p_{m} G\left(u_{n}, u_{m}\right)\right]^{D / 2-2} \\
C_{D} & \equiv \int_{0}^{1} d u_{4} \int_{0}^{u_{4}} d u_{3} \int_{0}^{u_{3}} d u_{2} \int_{0}^{u_{2}} d u_{1} \delta\left(u_{2}-u_{1}\right) \delta\left(u_{4}-u_{3}\right)\left[\sum_{n=1}^{4} \sum_{m=n+1}^{4} p_{n} \cdot p_{m} G\left(u_{n}, u_{m}\right)\right]^{D / 2-2} \\
D_{D} & \equiv \int_{0}^{1} d u_{4} \int_{0}^{u_{4}} d u_{3} \int_{0}^{u_{3}} d u_{2} \int_{0}^{u_{2}} d u_{1} \delta\left(u_{3}-u_{2}\right)\left[\sum_{n=1}^{4} \sum_{m=n+1}^{4} p_{n} \cdot p_{m} G\left(u_{n}, u_{m}\right)\right]^{D / 2-2}
\end{aligned}
$$

One trivially finds $A_{4}=\frac{1}{6}, B_{4}=\frac{3}{4}, C_{4}=\frac{1}{2}$ and $D_{4}=\frac{1}{4}$.

The remaing step is to perform all reorderings of the $u$ variables and divide by 4 !. In this way one arrives at the final expression

$$
\begin{aligned}
& \Gamma_{1}^{(M=4)}\left(p_{1}, p_{2}, p_{3}, p_{4}\right)=-\frac{1}{2}(2 \pi)^{4} \delta^{(4)}\left(p_{1}+p_{2}+p_{3}+p_{4}\right) \operatorname{Tr}_{C}\left(t_{G}^{a_{4}} t_{G}^{a_{3}} t_{G}^{a_{2}} t_{G}^{a_{1}}\right) \frac{g_{D}^{4}}{(2 \pi)^{D / 2}} \\
& \times\left\{4(D-2) A_{D}\left(\varepsilon^{1} \cdot \varepsilon^{2} \varepsilon^{3} \cdot \varepsilon^{4}+\varepsilon^{1} \cdot \varepsilon^{3} \varepsilon^{2} \cdot \varepsilon^{4}+\varepsilon^{1} \cdot \varepsilon^{4} \varepsilon^{2} \cdot \varepsilon^{3}\right)-2(D-2)\right. \\
& \times\left(B_{D}-C_{D}+D_{D}\right)\left(\varepsilon^{1} \cdot \varepsilon^{2} \varepsilon^{3} \cdot \varepsilon^{4}+\varepsilon^{1} \cdot \varepsilon^{4} \varepsilon^{2} \cdot \varepsilon^{3}\right)+16 C_{D}\left(\varepsilon^{1} \cdot \varepsilon^{2} \varepsilon^{3} \cdot \varepsilon^{4}+\varepsilon^{1} \cdot \varepsilon^{4} \varepsilon^{2} \cdot \varepsilon^{3}\right) \\
& \left.-32 C_{D} \varepsilon^{1} \cdot \varepsilon^{3} \varepsilon^{2} \cdot \varepsilon^{4}\right\} \Gamma\left(2-\frac{D}{2}\right)+f . t .
\end{aligned}
$$

which is in full agreement with the known results. 


\section{Concluding comments}

Given the schemes pioneered by Bern and Kosower [6] and reformulated by Strassler [13] based on string and world-line agents, respectively, and which aim at expediting perturbative computations in QCD both economically and efficiently, it becomes important to assess the relevant merits of yet another competitive proposal advanced in the present paper which utilizes the Polyakov world-line path integral. Directing, to begin with, our comments towards making comparisons with Strassler's approach we could say that the basic difference between the two world-line based schemes is how the disentanglement, between the weight factor pertaining to the spin of the propagating particle-like object on a given path and the dynamical factor represented by the Wilson line (loop), is accomplished. In Strassler's case this task is confronted by using super-particle degrees of freedom (one dimensional) and generates a term in the corresponding Lagrangian of the form $\psi^{\mu} F_{\mu \nu} \psi^{\nu}$. In the Polyakov (world-line) version, on the other hand, the issue is addressed via the introduction of the spin-factor. We believe that the separation, featured by the latter scheme, between 'geometrical' characteristics of paths on the one hand and dynamics -as embodied in the Wilson line(loop) factor- on the other, leads to an organization of the path integral expression which further facilitates the 'efficiency factor' for performing perturbative computations. In particular, it offers a unified base for treating, spinors, gauge fields and ghosts; all one has to do is adjust the master formula, which yields the computational rules, to the appropriate spin factor. Moreover, it lends itself to straightforward extensions for applications to processes involving open fermionic world-lines, as established in Refs [30, 31]. Referring, finally, to the stringbased approach of Bern and Kosower we note that the pinching issue, which arises in the non-abelian case and whose confrontation requires the application of a certain set of mnemonic rules, does not arise in our approach. We expect to further demostrate the virtues of the Polyakov world-line path integral scheme toward the calculation of two gluon loop contributions to the effective action by generating the corresponding master formulas.

\section{Acknowledgements}

One of us (S. D. A) acknowledges financial support from the Greek State Scholarships Foundation (I.K.Y.). A. I. K. and C. N. K. acknowledge the support from the General Secretariat of Research and Technology of the University of Athens. 


\section{Appendix}

In this Appendix we shall pay closer attention to the spin factor with respect to both carrying out the path integral in Eq. (3.3) and establishing the result encoded in Eqs. (3.14) and (3.15). Looking at the identity given by Eq. (2.11) we present the proper (regularized) expression for the tensor $\omega_{\mu \nu}$ reads as follows

$$
\int_{0}^{T} d t \omega_{\mu \nu}[\dot{x}(t)]=\lim _{\varepsilon \rightarrow 0} \frac{1}{4} \int_{-\varepsilon}^{\varepsilon} d s \int_{0}^{T} d t_{2} \int_{0}^{T} d t_{1}\left[\ddot{x}_{\mu}\left(t_{2}\right) \dot{x}_{\nu}\left(t_{1}\right)-\ddot{x}_{\nu}\left(t_{2}\right) \dot{x}_{\mu}\left(t_{1}\right)\right] \delta\left(t_{2}-t_{1}-s\right) .
$$

Now, if the functions (on the line) $x_{\mu}(t)$ are infinitely differentiable, then we can, once taking into account that $\left|t_{2}-t_{1}\right|<\varepsilon$, write $\ddot{x}_{\mu}\left(t_{2}\right)=\ddot{x}_{\mu}\left(t_{1}\right)+\mathcal{O}(s)$ as well as $\dot{x}_{\nu}\left(t_{1}\right)=$ $\dot{x}_{\nu}\left(t_{2}\right)+\mathcal{O}(s)$ and immediately conclude that

$$
\int_{0}^{T} d t \omega_{\mu \nu}[\dot{x}(t)]=\frac{T}{2} \int_{0}^{T} d t\left[\ddot{x}_{\mu}(t) \dot{x}_{\nu}(t)-\ddot{x}_{\nu}(t) \dot{x}_{\mu}(t)\right]
$$

Otherwise, one should use the limiting expression according to (A.1) when performing manipulations that involve the spin factor.

Let us proceed with the computation of the path integral entering Eq. (3.3). We set

$$
\mathrm{I}_{\mu \nu}=\int d^{4} a \int_{x(0)=x(T)=a} D x(t) \Phi^{[1]}[\dot{x}(t)]_{\mu \nu} \exp \{-S[x]\}
$$

where

$$
S[x]=\frac{1}{4} \int_{0}^{T} d t \dot{x}^{2}(t)-i \sum_{n=1}^{M} \hat{k}\left(t_{n}\right) \cdot x\left(t_{n}\right)
$$

To compute $\mathrm{I}_{\mu \nu}$ we make the variable change $x \rightarrow x+x^{c l}$, where $x^{c l}$ is a solution of the classical equation of motion resulting from the above action. Specifically, we have

$$
\ddot{x}_{\mu}^{c l}(t)=-2 i \sum_{n=1}^{M} \hat{k}\left(t_{n}\right) \delta\left(t-t_{n}\right) \Rightarrow x_{\mu}^{c l}(t)=2 i \sum_{n=1}^{M} \hat{k}\left(t_{n}\right) \Delta\left(t, t_{n}\right)+a,
$$

where we have employed the Green's function

$$
\Delta\left(t, t^{\prime}\right)=\frac{t\left(T-t^{\prime}\right)}{T} \theta\left(t^{\prime}-t\right)+\frac{t^{\prime}(T-t)}{T} \theta\left(t-t^{\prime}\right), \quad \Delta\left(0, t^{\prime}\right)=\Delta\left(T, t^{\prime}\right)=0 .
$$


The new action functional is now specified by

$$
S[x] \rightarrow \frac{1}{4} \int_{0}^{T} d t \dot{x}^{2}(t)+S\left[x^{c l}\right]
$$

where

$$
S\left[x^{c l}\right]=\sum_{n=1}^{M} \sum_{m=1}^{M} \hat{k}\left(t_{n}\right) \cdot \hat{k}\left(t_{m}\right) \Delta\left(t_{n}, t_{m}\right)-i \sum_{n=1}^{M} p_{n} \cdot a .
$$

We immediately observe that integration over $\alpha$ (translational zero modes) leads to momentum conservation which enters Eq. (3.7). The rest of the expression for $S\left[x^{c l}\right]$ produces the terms entering the exponential factor in the same equation.

Turning our attention to the spin factor we first note that the variable change $x \rightarrow$ $x+x^{c l}$ leads to

$$
\int_{0}^{T} d t \omega_{\mu \nu}[\dot{x}] \rightarrow \int_{0}^{T} d t \omega_{\mu \nu}\left[\dot{x}^{c l}\right]+\frac{T}{2} \int_{0}^{T} d t\left[\ddot{x}_{\mu}(t) \dot{x}_{\nu}(t)-\ddot{x}_{\nu}(t) \dot{x}_{\mu}(t)\right],
$$

having taken into account that the contours $x(t)$ are to be integrated with respect to a quadradtic action functional (cf. Eq. (A.7)), which implies, cf. Eqs. (A.1) and (A.5), that mixed terms in $x$ and $x^{c l}$ drop out. Let us finally note that for paths that are infinitely differentiable, in which case Eq. (A.2) strictly holds true, the integration of the spin factor with respect to the quadratic action functional yields unity. All this leads to the following result as far as performing the path integral in Eq. (A.3) is concerned.

$$
\begin{aligned}
& I_{\mu \nu}=(2 \pi)^{D} \delta^{(D)}\left(\sum_{n=1}^{M} p_{n}\right) \frac{1}{(4 \pi T)^{D / 2}} \Phi^{[1]}\left[\dot{x}^{c l}\right]_{\mu \nu} \\
& \times \exp \left[\sum_{n<m} p_{n} \cdot p_{m} G\left(t_{n}, t_{m}\right)+\sum_{n \neq m} \bar{\xi}_{n} \xi_{n} \varepsilon^{n} \cdot p_{m} \partial_{n} G\left(t_{n}, t_{m}\right)\right. \\
& \left.\quad+\frac{1}{2} \sum_{n \neq m} \bar{\xi}_{n} \xi_{n} \bar{\xi}_{m} \xi_{m} \varepsilon^{n} \cdot \varepsilon^{m} \partial_{n} \partial_{m} G\left(t_{n}, t_{m}\right)\right] .
\end{aligned}
$$

The above result explicitly demonstrates our assertion that the overall contribution from the spin factor is exclusively determined by those points on a given path where a momentum is imparted via the action of an external gauge field.

The final result is obtained once we substitute (A.5) into (A.1). We get

$$
\begin{aligned}
\int_{0}^{T} d t \omega_{\mu \nu}\left[\dot{x}^{c l}\right]=- & 2 \sum_{n=1}^{M} \bar{\xi}_{n} \xi_{n}\left(\varepsilon_{\mu}^{n} p_{n, \nu}-\varepsilon_{\nu}^{n} p_{n, \mu}\right)+\sum_{n=0}^{M} \sum_{m=0}^{M} \bar{\xi}_{n} \xi_{n} \bar{\xi}_{m} \xi_{m}\left(\varepsilon_{\mu}^{n} \varepsilon_{\nu}^{m}-\varepsilon_{\nu}^{n} \varepsilon_{\mu}^{m}\right) \\
& \times \int_{-\varepsilon}^{\varepsilon} d s \frac{\partial}{\partial t_{n}} \delta\left(t_{n}-t_{m}-s\right) .
\end{aligned}
$$


The correct handling of the last term follows once we take into consideration that, first, $m \neq n$ on account of the Grassmann variables and, second, it is to be integrated in consistence with the time ordering implicit in Eq. (3.1) of the text. Specifically, we have

$$
\begin{aligned}
& \ldots \int_{0}^{T} d t_{n+1} \int_{0}^{T} d t_{n} \int_{0}^{T} d t_{n-1} \theta\left(t_{n+1}-t_{n}\right) \theta\left(t_{n}-t_{n-1}\right) \int_{-\varepsilon}^{\varepsilon} d s \frac{\partial}{\partial t_{n}} \delta\left(t_{n}-t_{m}-s\right) \ldots= \\
= & \ldots \int_{0}^{T} d t_{n+1} \int_{0}^{T} d t_{n} \int_{0}^{T} d t_{n-1} \theta\left(t_{n+1}-t_{n}\right) \theta\left(t_{n}-t_{n-1}\right) \\
& \times\left[2 \delta_{n+1, m} \delta\left(t_{n+1}-t_{n}\right)-2 \delta_{m, n-1} \delta\left(t_{n}-t_{n-1}\right)\right] \ldots,
\end{aligned}
$$

which allows us to return to Eq. (A.11) and infer that

$$
\begin{array}{r}
\int_{0}^{T} d t \omega_{\mu \nu}\left[\dot{x}^{c l}\right]=-\frac{i}{2} \int_{0}^{T} d t\left(J \cdot \omega^{c l}\right)_{\mu \nu}=-2 \sum_{n=0}^{M} \bar{\xi}_{n} \xi_{n}\left(\varepsilon_{\mu}^{n} p_{n, \nu}-\varepsilon_{\nu}^{n} p_{n, \mu}\right)- \\
\quad-4 \sum_{n=1}^{M} \bar{\xi}_{n+1} \xi_{n+1} \bar{\xi}_{n} \xi_{n}\left(\varepsilon_{\mu}^{n+1} \varepsilon_{\nu}^{n}-\varepsilon_{\nu}^{n+1} \varepsilon_{\mu}^{n}\right) \delta\left(t_{n+1}-t_{n}\right)
\end{array}
$$

With the above results in place, Eqs. (3.14) and (3.15) in the text follow directly.

The careful course of reasoning we have followed in this appendix can be circumvented by the more formal line of procedure adopted in the text. Thus, the validity of the aformentioned equations can be established once we observe that

$$
\begin{aligned}
& \exp \left[-i \sum_{n=1}^{M} \hat{k}\left(t_{n}\right) \cdot x\left(t_{n}\right)\right] \int_{0}^{T} d t \frac{\delta}{\delta s_{\mu \nu}(t)} \exp \left[i \sum_{n=1}^{M} \hat{k}\left(t_{n}\right) \cdot x\left(t_{n}\right)\right] \\
& =-\lim _{\varepsilon \rightarrow 0} \int_{0}^{T} d t \sum_{n=1}^{M} \sum_{m=1}^{M} \int_{-\varepsilon}^{\varepsilon} d s s \hat{k}_{\mu}\left(t_{n}\right) \delta\left(t_{n}-t-\frac{s}{2}\right) \hat{k}_{\nu}\left(t_{m}\right) \delta\left(t_{m}-t+\frac{s}{2}\right) \\
& =\frac{1}{4} \lim _{\varepsilon \rightarrow 0} \int_{0}^{T} d t \int_{-\varepsilon}^{\varepsilon} d s s \ddot{x}_{\mu}^{c l}\left(t+\frac{s}{2}\right) \ddot{x}_{\nu}^{c l}\left(t-\frac{s}{2}\right) \\
& =\lim _{\varepsilon \rightarrow 0} \frac{1}{8} \int_{-\varepsilon}^{\varepsilon} d s \int_{0}^{T} d t_{2} \int_{0}^{T} d t_{1}\left[\ddot{x}_{\mu}\left(t_{2}\right) \dot{x}_{\nu}\left(t_{1}\right)-\ddot{x}_{\nu}\left(t_{2}\right) \dot{x}_{\mu}\left(t_{1}\right)\right] \delta\left(t_{2}-t_{1}-s\right) .
\end{aligned}
$$

\section{References}

[1] R. R. Metsaev and A. A. Tseytlin, Nucl. Phys. B 298, 109 (1988). 
[2] J. A. Minahan, Nucl. Phys. B 298, 36 (1988).

[3] T. R. Taylor and G. Veneziano, Phys. Lett. B 212, 147 (1988).

[4] V. S. Kaplunovsky, Nucl. Phys. B 307, 145 (1988) [Erratum-ibid. B 382, 436 (1988)] arXiv:hep-th/9205068.

[5] Z. Bern and D. A. Kosower, Phys. Rev. D 38, 1888 (1988).

[6] Z. Bern and D. A. Kosower, Nucl. Phys. B 321, 605 (1989), and Nucl. Phys. B 379, 451 (1992).

[7] Z. Bern, L. J. Dixon and D. A. Kosower, Phys. Rev. Lett. 70, 2677 (1993) arXiv:hep-ph/9302280.

[8] Z. Bern, Phys. Lett. B 296, 85 (1992).

[9] Z. Bern and D. C. Dunbar, Nucl. Phys. B 379, 562 (1992).

[10] P. Di Vecchia, A. Lerda, L. Magnea and R. Marotta, Phys. Lett. B 351, 445 (1995) arXiv:hep-th/9502156].

[11] E. S. Fradkin and A. A. Tseytlin, Phys. Lett. B 163, 123 (1985).

[12] P. Di Vecchia, L. Magnea, A. Lerda, R. Russo and R. Marotta, Nucl. Phys. B 469, 235 (1996) arXiv:hep-th/9601143.

[13] M. J. Strassler, Nucl. Phys. B 385, 145 (1992) arXiv:hep-ph/9205205.

[14] R. Casalbuoni, J. Gomis and G. Longhi, Nuovo Cim. A 24, 249 (1974).

[15] L. Brink, S. Deser, B. Zumino, P. Di Vecchia and P. Howe, Phys. Lett. B 64, 435 (1976), and L. Brink, P. Di Vecchia and P. Howe, Nucl. Phys. B 118, 76 (1977).

[16] A. P. Balachandran, P. Salomonson, B. S. Skagerstam and J. O. Winnberg, Phys. Rev. D 15, 2308 (1977).

[17] M. G. Schmidt and C. Schubert, Phys. Lett. B 331, 69 (1994) arXiv:hepth/9403158].

[18] M. G. Schmidt and C. Schubert, Phys. Rev. D 53, 2150 (1996) arXiv:hepth/9410100].

[19] M. Reuter, M. G. Schmidt and C. Schubert, Annals Phys. 259, 313 (1997) arXiv:hep-th/9610191.

[20] V. A. Fock, Izv. Akad. Nauk. USSR, OMEN, 557 (1937).

[21] R. P. Feynman, Phys. Rev. 80, 440 (1950). 
[22] J. S. Schwinger, Phys. Rev. 82, 664 (1951).

[23] E. S. Fradkin, Nucl. Phys. B 76, 588 (1966) .

[24] M. B. Halpern, A. Jevicki and P. Senjanovic, Phys. Rev. D 16, 2476 (1977), and M. B. Halpern and W. Siegel, Phys. Rev. D 16, 2486 (1977).

[25] L. Alvarez-Gaume, Commun. Math. Phys. 90, 161 (1983).

[26] A. M. Polyakov in: E. . Brezin and J. . Zinn-Justin, "Fields, Strings And Critical Phenomena" Amsterdam, Netherlands: North-Holland (1990).

[27] A. I. Karanikas and C. N. Ktorides, Phys. Lett. B 275, 403 (1992).

[28] A. I. Karanikas and C. N. Ktorides, Phys. Rev. D 52, 5883 (1995).

[29] G. C. Gellas, A. I. Karanikas and C. N. Ktorides, Annals Phys. 255, 228 (1997); Phys. Rev. D 55, 5027 (1997); Phys. Rev. D 57, 3763 (1998); A. I. Karanikas and C. N. Ktorides, Phys. Rev. D 59, 016003 (1999) arXiv:hep-ph/9807385; A. I. Karanikas, C. N. Ktorides, N. G. Stefanis and S. M. Wong, Phys. Lett. B 455, 291 (1999) [arXiv:hep-ph/9812335].

[30] A. I. Karanikas and C. N. Ktorides, JHEP 9911, 033 (1999) [arXiv:hep-th/9905027.

[31] A. I. Karanikas and C. N. Ktorides, Phys. Lett. B 500, 75 (2001) arXiv:hepth/0008078].

[32] A. S. Kapoyannis, A. I. Karanikas and C. N. Ktorides, in preparation.

[33] A. M. Polyakov, Nucl. Phys. B 164, 171 (1980).

[34] A. A. Migdal, Phys. Rept. 102, 199 (1983). 